\title{
On the number of radial orderings of planar point sets
}

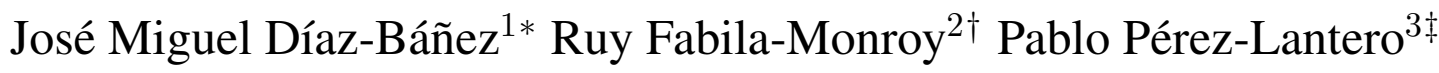 \\ ${ }^{1}$ Departamento Matemática Aplicada II. Universidad de Sevilla. Spain \\ ${ }^{2}$ Departamento de Matemáticas. Centro de Investigación y de Estudios Avanzados del Instituto Politécnico Nacional. Mexico \\ ${ }^{3}$ Escuela de Ingeniería Civil Informática. Universidad de Valparaíso. Chile
}

received $16^{\text {th }}$ Apr. 2012, revised $28^{\text {th }}$ June 2013, accepted $9^{\text {th }}$ Dec. 2014.

Given a set $S$ of $n$ points in the plane, a radial ordering of $S$ with respect to a point $p$ (not in $S$ ) is a clockwise circular ordering of the elements in $S$ by angle around $p$. If $S$ is two-colored, a colored radial ordering is a radial ordering of $S$ in which only the colors of the points are considered. In this paper, we obtain bounds on the number of distinct non-colored and colored radial orderings of $S$. We assume a strong general position on $S$, not three points are collinear and not three lines—each passing through a pair of points in $S$-intersect in a point of $\mathbb{R}^{2} \backslash S$. In the colored case, $S$ is a set of $2 n$ points partitioned into $n$ red and $n$ blue points, and $n$ is even. We prove that: the number of distinct radial orderings of $S$ is at most $O\left(n^{4}\right)$ and at least $\Omega\left(n^{3}\right)$; the number of colored radial orderings of $S$ is at most $O\left(n^{4}\right)$ and at least $\Omega(n)$; there exist sets of points with $\Theta\left(n^{4}\right)$ colored radial orderings and sets of points with only $O\left(n^{2}\right)$ colored radial orderings.

Keywords: radial orderings, colored point sets, star polygonizations

\section{Introduction}

Let $S$ be a set of $n$ points in the plane. We say that $S$ is in strong general position if it is ineneral position (not three of its points are collinear) and every time that three lines-each passing through a pair of points in $S$-intersect, they do so in a point in $S$. Unless otherwise noted, all point sets in this paper are in strong general position. Let $p$ be a point not in $S$ such that $S \cup\{p\}$ is in general position; we call $p$ an observation point. A radial ordering of $S$ with respect to $p$ is a clockwise circular ordering of the points in $S$ by their angle around $p$. Thus these orderings are equivalent under rotations. If every point in $S$ is assigned one of two colors, say red and blue, then a colored radial ordering of $S$ with respect to $p$ is a circular clockwise ordering of the colors of the points in $S$ by their angle around $p$. Thus permutations between points of the same color yield the same colored radial ordering.

\footnotetext{
*Partially supported by projects FEDER P09-TIC-4840, and by the ESF EUROCORES program EuroGIGA-ComPoSe IP04MICINN Project EUI-EURC-2011-4306.

${ }^{\dagger}$ Partially supported by Conacyt of Mexico, grant 153984.

¥Partially supported by projects CONICYT FONDECYT/Iniciación 11110069 (Chile), and Millennium Nucleus Information and Coordination in Networks ICM/FIC P10-024F (Chile).
}

1365-8050 @ 2014 Discrete Mathematics and Theoretical Computer Science (DMTCS), Nancy, France 
Let $\rho(S)$ be the number of distinct radial orderings of $S$ with respect to every observation point in the plane. Likewise, let $\rho_{\text {col }}(S)$ be the number of distinct colored radial orderings of $S$ with respect to every observation point in the plane. We define the following functions:

$$
\begin{aligned}
f(n) & :=\max \{\rho(S): S \text { is a set of } n \text { points }\} \\
f_{\text {col }}(n) & :=\max \left\{\rho_{\text {col }}(S): n \text { is even and } S \text { is a set of } n \text { red and } n \text { blue points }\right\} \\
g(n) & :=\min \{\rho(S): S \text { is a set of } n \text { points }\} \\
g_{\text {col }}(n) & :=\min \left\{\rho_{\text {col }}(S): n \text { is even and } S \text { is a set of } n \text { red and } n \text { blue points }\right\}
\end{aligned}
$$

In this paper we prove the following bounds.

$$
\begin{aligned}
f(n) & =\Theta\left(n^{4}\right) \\
f_{\text {col }}(n) & =\Theta\left(n^{4}\right) \\
\Omega\left(n^{3}\right) \leq g(n) \leq & \leq O\left(n^{4}\right) \\
\Omega(n) \leq g_{\text {col }}(n) & \leq O\left(n^{2}\right)
\end{aligned}
$$

The first equality $\left(f(n)=\Theta\left(n^{4}\right)\right)$ has been noted before in the literature. In [8, 7, 15, 16] $f(n)=$ $O\left(n^{4}\right)$ is proved. In [16] the author proves both $f(n)=O\left(n^{4}\right)$ and $f(n)=\Omega\left(n^{4}\right)$. As far as we know, all the other bounds are new.

A different problem but in the same setting has been studied recently in [15]. In that paper, the authors study what a robot can infer from its environment when all the information that is available is the cyclic positions of some landmarks as they appear from the robot's position. Other authors have considered problems of the same flavor, when a similar kind of information is available. See for example [10, 11, 14]. In [8] the authors study the algorithmic problem of updating the radial ordering of a moving observation point.

We point out that computing the radial ordering of $S$ around every point in $S$ is an unavoidable step in some geometric algorithms, as for example, performing a radial sweeping of a point set. Moreover, many optimization problems are solved by considering the arrangement generated by every line passing through every pair of points in $S$, and finding the optimum point inside each of the $O\left(n^{4}\right)$ cells in the arrangement [12]. In many cases this is because the radial ordering of the points in $S$ around every point within a cell is the same. It could be interesting in this scenario to know how many cells induce the same radial ordering.

For a bi-colored point set, a radial sweeping algorithm also requires the ordering as an initial step, so it could be useful to know bounds on the number of different colored radial ordering of $S$ from points in the plane. From the combinatorial point of view, this problem is related to partitioning bi-colored point sets with $k$-fans [3, 4]. A $k$-fan in the plane is a point $p$ (called the center) and $k$ rays emanating from $p$. This structure can be used to partition $S$ into $k$ monochromatic subsets and it depends only on the colored radial ordering of $S$ with respect to $p$. The existence of balanced-each part having an equal number of red an blue points - $k$-fans for colored point sets has been studied in recent papers [5, 6] but, as far as we know, the number of different monochromatic partitions induced by $k$-fans has not yet been considered.

The assumptions that $S$ is in strong general position; that $S$ has the same number of red and blue points and that $n$ is even, may seem arbitrary. However, the three of them are crucial hypothesis in our results (see Section 4).

A preliminary version of this paper appeared in [9]. 


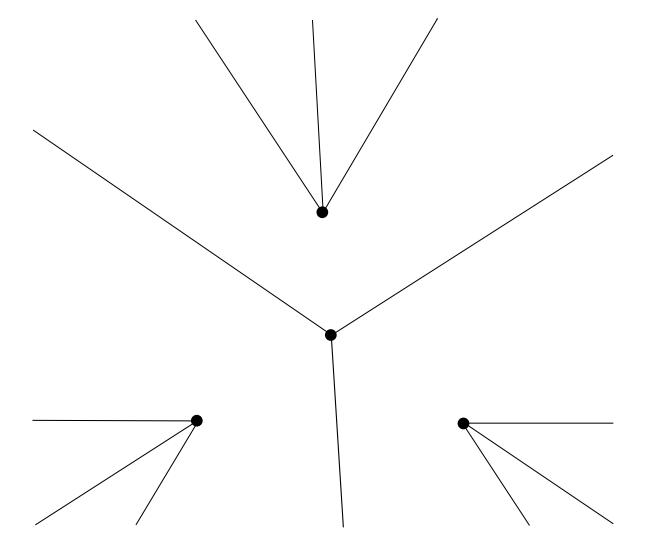

Fig. 1: The order partition of a set of four points.

\section{Uncolored Case}

In this section $S$ is a set of $n$ points in strong general position in the plane. We discretize the problem by partitioning the set of observation points into a finite number of sets so that points in the same set induce the same radial ordering. This partition is made by half-lines, which if crossed by an observation point, generate a transposition of two consecutive elements in the radial ordering. For every pair of points $x_{1}, x_{2} \in S$, consider the line passing through them. Contained in this line we have two half-lines; one begins in $x_{1}$ and does not contain $x_{2}$, while the other begins in $x_{2}$ and does not contain $x_{1}$. Two observation points are in the same element of the partition if they can be connected by a path which does not intersect any half-line. We call this partition the order partition (see Figure 11. Since it induces a decomposition of the plane, we refer to its elements as cells. The order partition is used (under different names) also in [8, 7, 15]. Note that if a point moves in a path not crossing any half-line, the radial ordering with respect to this point is the same throughout the motion. Thus points in the same cell induce the same radial ordering. As a set of three points already shows, the converse is not true in general; two observation points may lie in different cells of the order partition and induce the same radial ordering of $S$.

As mentioned before, the following two bounds on $f(n)$ have been proved before; we provide proofs for completeness.

Theorem 2.1 $f(n) \leq O\left(n^{4}\right)$.

Proof: The order partition cannot have more cells than the arrangement induced by the lines passing through each pair of points in $S$. Such an arrangement has $O\left(n^{4}\right)$ cells.

Theorem $2.2 f(n) \geq \Omega\left(n^{4}\right)$.

Proof: Follows from $f(n) \geq f_{\text {col }}(n)$ and Theorem 3.2

We now prove an upper and a lower bound on $g(n)$. The upper bound follows from the upper bound on $f(n)$.

Theorem 2.3 $g(n) \leq O\left(n^{4}\right)$. 
The lower bound on $g(n)$ is far more elaborate. First we show a lower bound of $\Omega\left(n^{4}\right)$ on the number of cells in the order partition. As far as we know, this is the first lower bound ever given on the size of the order partition.

Theorem 2.4 The number of cells in the order partition of $S$ is $\Omega\left(n^{4}\right)$.

Proof: Let $\mathcal{L}$ be the set of lines passing through each pair of points in $S$. First we show that in the line arrangement $\mathcal{A}$ generated by $\mathcal{L}$ there are $\frac{n^{4}}{8}-O\left(n^{3}\right)$ cells. We may regard $\mathcal{A}$ as a plane graph $G$ with an extra vertex $v^{*}$ placed at infinity. So that $v^{*}$ is contained in all the unbounded faces of $G$ and twice in all the lines in $\mathcal{L}$. Let $V, E$ and $F$ be the number of vertices, edges and faces of $G$ respectively. Note that by our strong general assumption $G$ has exactly: one vertex of degree $n(n-1)$; $n$ vertices of degree $2(n-1)$ and $V-n-1$ vertices of degree 4 . Thus $E$ equals $n(n-1) / 2+n(n-1)+2 V-2 n-2$ which is $2 V+O\left(n^{2}\right)$. By Euler's formula $F$ equals $V+O\left(n^{2}\right)$. Let $p$ and $q$ be any two points in $S$. Let $L_{p}$ be the set of lines in $\mathcal{L}$ that contain $p$ but not $q$. Let $L_{q}$ be the set of lines in $\mathcal{L}$ that contain $q$ but not $p$. Note that each line in $L_{p}$ intersects each line in $L_{q}$ in a point not in $S$, with the exception of only two cases. The case in which both lines are parallel or the case in which they both contain the same point in $S \backslash\{p, q\}$. In total each case occurs at most $(n-2)$ times; thus $p$ and $q$ induce at least $(n-2)(n-2)-2(n-2)=(n-2)(n-4)$ vertices of degree 4 . Doing this for every pair of vertices, we count each 4-degree vertex exactly four times. In total there are $\frac{n^{4}}{8}-O\left(n^{3}\right)$ vertices and $\frac{n^{4}}{8}-O\left(n^{3}\right)$ faces in $G$ (and the same number of cells in $\mathcal{A})$. The order partition can be obtained by removing from $\mathcal{A}$ each line segment joining a pair of points of $S$. Let $e_{1}, \ldots, e_{\left(\begin{array}{c}n \\ 2\end{array}\right)}$ be these line segments in any given order. Let $\operatorname{cr}(S)$ be the number of pairs of these edges that intersect in their interior. Let $M$ be the number of vertices of $\mathcal{A}$ lying in the interior of any of the $e_{i}$ 's. Note that for each set of four points of $S$ we obtain: one vertex of $M$ if the set is in convex position and three vertices otherwise. The number of sets of four elements of $S$ that are in convex position is precisely $\operatorname{cr}(S)$. Thus $M=3\left(\begin{array}{c}n \\ 4\end{array}\right)-2 \operatorname{cr}(S)$. It is known that $\operatorname{cr}(S)$ is bounded from below by $\frac{3}{8}\left(\begin{array}{c}n \\ 4\end{array}\right)$ (see [1, 2, 13]). Thus $M$ is at most $\frac{9}{4}\left(\begin{array}{c}n \\ 4\end{array}\right)=\frac{3}{32} n^{4}-O\left(n^{3}\right)$. We remove each $e_{i}$ in order, and show that at the end $\Omega\left(n^{4}\right)$ cells remain. Let $d_{i}$ be the number of vertices in $M$ lying in the interior of $e_{i}$ just before it is removed. Note that when $e_{i}$ is removed, in the worst case, $d_{i}+1$ cells of $\mathcal{A}$ are lost. Thus when all of the $e_{i}$ 's are removed at least $\frac{n^{4}}{8}-O\left(n^{3}\right)-\sum_{i=1}^{\left(\begin{array}{c}n \\ 2\end{array}\right)}\left(d_{i}+1\right)=\frac{n^{4}}{8}-O\left(n^{3}\right)-M-\left(\begin{array}{c}n \\ 2\end{array}\right) \geq \frac{n^{4}}{32}-O\left(n^{3}\right)$ cells remain.

We now prove a useful lemma for finding distinct radial orderings of $S$.

Lemma 2.5 (Partition Lemma) Let $(R, B)$ be a partition of $S$ into non-empty subsets. Let $p$ and $q$ be two points in different cells of the order partition. If no half-line spanned by a point in $R$ and point in $B$ intersects the line segment with endpoints $p$ and $q$, then the radial orderings of $S$ as seen from $p$ and $q$ are distinct.

Proof: Since $p$ and $q$ lie in different cells of the order partition, the line segment joining them must intersect at least one half-line. Let $x_{1}, x_{2} \in S$ be the points defining this half-line. Note that $x_{1}$ and $x_{2}$ are both in $R$ or both in $B$. Let $x_{3} \in S$ be a point in the element of the partition not containing $x_{1}$ and $x_{2}$. Assume without loss of generality that the radial ordering of $\left\{x_{1}, x_{2}, x_{3}\right\}$ with respect to $p$ is $\left[x_{1}, x_{2}, x_{3}\right]$. Since the line segment joining $p$ and $q$ crosses the half-line only once, the radial ordering of $\left\{x_{1}, x_{2}, x_{3}\right\}$ with respect to $q$ is $\left[x_{2}, x_{1}, x_{3}\right]$. Therefore, the radial ordering of $S$ with respect to $p$ is different from the radial ordering with respect to $q$. 


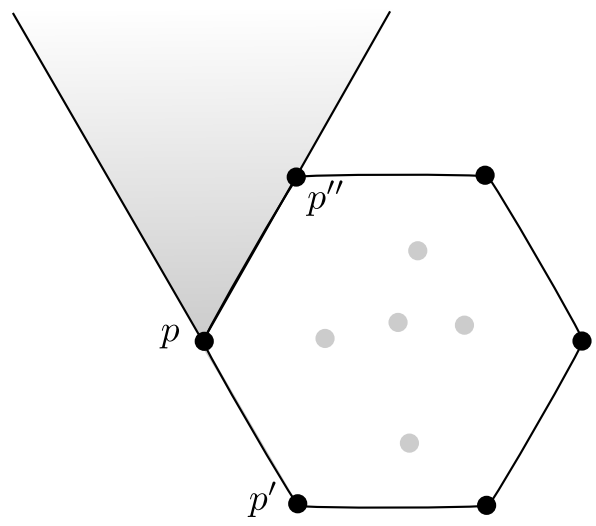

Fig. 2: The cone in the proof of Theorem 2.7

The following Lemma is also proved in [7](Theorem 4.2) and in [15](Theorem 1), we include a proof for completeness.

Lemma 2.6 Let $p$ and $q$ be two observation points in the interior of the convex hull of $S$, lying in different cells of the order partition. Then the radial orderings of $S$ with respect to $p$ and $q$ are distinct.

Proof: Let $\ell$ be the straight line containing $p$ and $q$. Since $p$ and $q$ are in the interior of the convex hull of $S, \ell$ partitions $S$ into two sets that together with $p$ and $q$ satisfy the conditions of the Partition Lemma.

Finally, we combine the above results to prove the lower bound on $g(n)$.

Theorem $2.7 g(n) \geq \Omega\left(n^{3}\right)$.

Proof: An open cell of the order partition is interior if it intersects the interior of the convex hull of $S$ and it is exterior if it has a point not in the interior of the convex hull of $S$. Note that a cell can be both interior and exterior. If less than half of the the cells are exterior then at least half of them are interior and we are done by Theorem 2.4 and Lemma 2.6 Assume then, that at least half of the cells are exterior. Thus there are $\Omega\left(n^{4}\right)$ exterior cells.

Let $C$ be the convex hull of $S$ and $m$ be its number of vertices. Let $p$ be one of these vertices. Let $p^{\prime}$ and $p^{\prime \prime}$ be the vertices previous and next to $p$ in $C$ in clockwise order respectively. Let $\Gamma_{p}$ be the convex cone with apex $p$, bounded by: the infinite ray with apex $p$ and passing through $p^{\prime \prime}$ and the infinite ray with apex $p^{\prime}$ and passing through $p$ (see Figure 2). Let $R:=\{p\}$ and $B:=S \backslash\{p\}$. Note that any two points in $\Gamma_{p}$ lying in different cells of the order partition, together with $R$ and $B$, satisfy the conditions of the Partition Lemma. Therefore, the radial orderings of $S$ with respect to any two points in $\Gamma_{p}$ lying in different cells of the order partition are distinct. For each vertex of $C$ define such a cone. Every exterior cell intersects one of these cones. Therefore there is a cone intersecting $\Omega\left(n^{4} / m\right)=\Omega\left(n^{3}\right)$ of them and the result follows. 


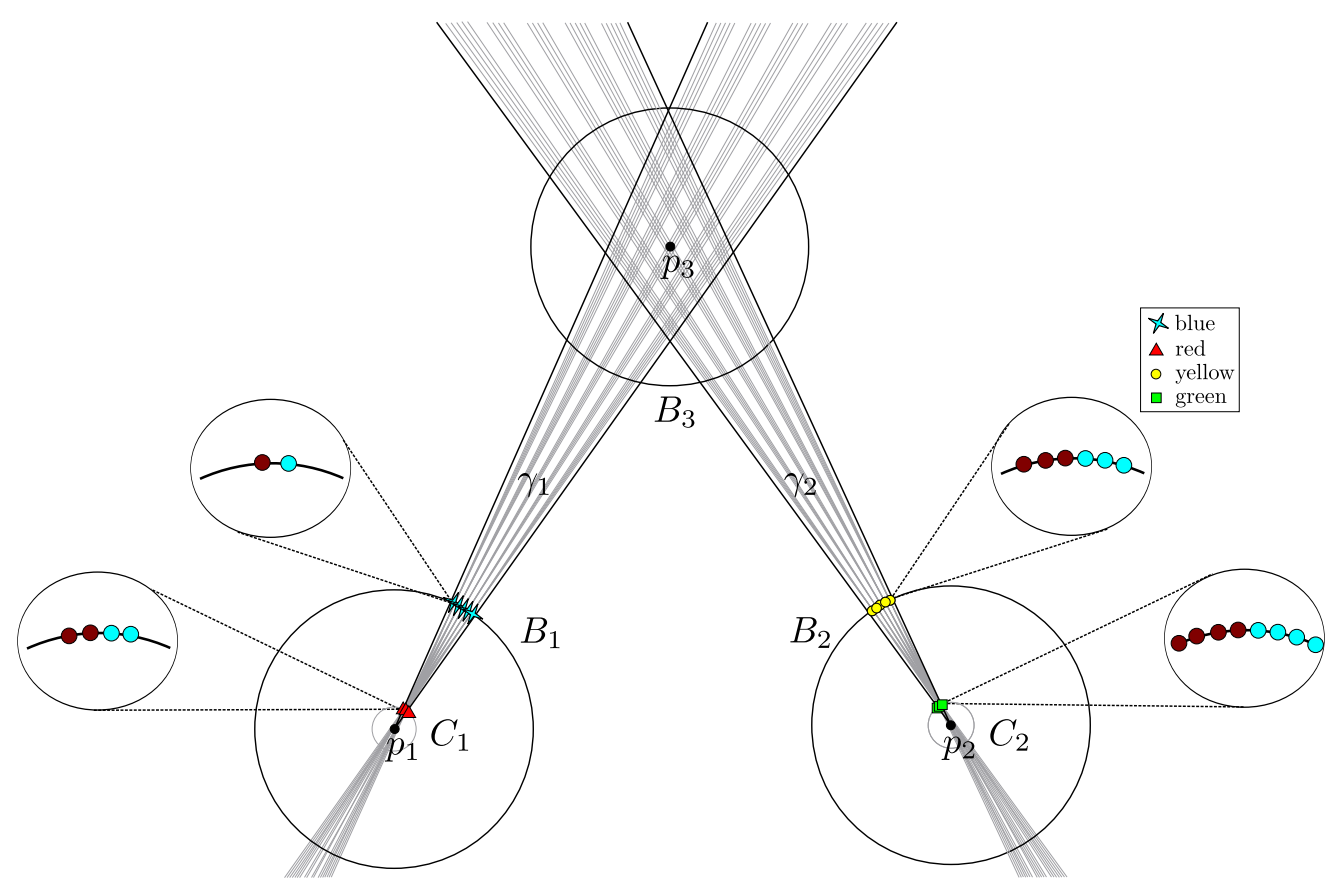

Fig. 3: A bi-colored set with $\Omega\left(n^{4}\right)$ different colored radial orderings.

\section{Colored Case}

In this section $n$ is even, and $S$ is a set of $n$ red and $n$ blue points in strong general position in the plane. We prove upper and lower bounds on $f_{\text {col }}(n)$ and $g_{\text {col }}(n)$.

Theorem $3.1 f_{\text {col }}(n) \leq O\left(n^{4}\right)$.

Proof: Follows from $f_{\text {col }}(n) \leq f(n)$ and Theorem 2.1

Theorem $3.2 f_{\text {col }}(n) \geq \Omega\left(n^{4}\right)$.

Proof: Assume that $n \geq 20$. We start by constructing a four-colored set of points $S^{\prime}$ with $\Omega\left(n^{4}\right)$ distinct colored radial orderings; afterwards we obtain $S$ by replacing each point of a given color with a suitable "pattern" of red and blue points. These four patterns are chosen so that if they appear consecutively in a radial ordering, then any other equivalent radial ordering must match patterns of the same type. Since the patterns behave like the original four colors, the new set also has $\Omega\left(n^{4}\right)$ colored radial orderings.

Let $B_{1}, B_{2}$ and $B_{3}$ be three disks of radius $1 / 4$, whose centers $p_{1}, p_{2}$, and $p_{3}$ are the vertices of an equilateral triangle of side length equal to one. Let $\varepsilon, \alpha>0$. Let $C_{1}$ and $C_{2}$ be circles of radius $\varepsilon$ centered at $p_{1}$ and $p_{2}$, respectively. Let $\gamma_{1}$ and $\gamma_{2}$ be infinite wedges of angle $\alpha$, with apices $p_{1}$ and $p_{2}$ respectively. Assume that $\gamma_{1}$ is bisected by the line segment joining $p_{1}$ and $p_{3}$, while $\gamma_{2}$ is bisected by the line segment joining $p_{2}$ and $p_{3}$. Refer to Figure 3 . Let $m$ and $r$ be the only natural numbers such that $n=10 m+r$ 
and $10 \leq r \leq 19$. Partition $\gamma_{1}$ with $m$ infinite rays emanating from $p_{1}$, such that the angle between two consecutive rays equals $\alpha /(m+1)$. Do likewise for $\gamma_{2}$, with $m$ infinite rays emanating from $p_{2}$. At every point of intersection of these rays with the boundary of $B_{1}$, place a blue point; at every point of intersection with $C_{1}$ a red point; at every point of intersection with the boundary of $B_{2}$ a yellow point; finally, at every point of intersection with $C_{2}$ a green point. Thus $m$ points of each color are placed. This ends the construction of $S^{\prime}$.

Let $L$ be the set of lines passing through a red and a blue point. Let $L^{\prime}$ be the set of lines passing through a yellow and a green point. Choose $\alpha$ and $\varepsilon$ small enough so that the following conditions are met: (1) Every line in $L \cup L^{\prime}$ intersects the interior of $B_{3}$ and these are the only lines passing through two points of $S^{\prime}$ that do. (2) Neither two lines in $L$ nor two lines in $L^{\prime}$ intersect at a point in the interior of $B_{3}$. (3) Every line in $L$ intersects every line in $L^{\prime}$ at a point in $B_{3}$. By the previous conditions and the fact that $|L|=m^{2}$ and $\left|L^{\prime}\right|=m^{2}, L \cup L^{\prime}$ splits $B_{3}$ into precisely $(m+1)^{4}$ cells. For each of these cells choose a point $q_{i}$ in its interior. We show that the colored radial orderings of $S^{\prime}$ as seen from each of these points is different. Note that for each point in $B_{3}$ there exists a line separating the red and blue points from the green and yellow points. Thus we may assume, that the colored radial orderings as seen from points in $B_{3}$ are written so that all the blue and red points appear before the green and yellow points. Let $q_{i}$ and $q_{j}$ be two points in $B_{3}$, belonging to different cells of the order partition. Consider the colored radial ordering when walking from $q_{i}$ to $q_{j}$ in a straight line. By conditions (1) and (2), the only transpositions that occur when a half-line is crossed is between a red and a blue point or between a yellow and a green point. This implies that the $k$-th red point is always the same red point and that the number of blue points after the $k$-th red point is either increasing or decreasing monotonically; the same observation holds for the green and yellow points. Therefore, in the walk once a line in $L \cup L^{\prime}$ is crossed, all colored radial orderings afterwards will be distinct. Thus the number of different colored radial orderings of $S^{\prime}$ is at least $(m+1)^{4}$, which is $\Omega\left(n^{4}\right)$.

To construct $S$, we replace the points in $S^{\prime}$ by patterns of red and blue points, in such a way that the colored radial orderings at points $q_{i}$ remain different. The points in the patterns replacing a point $p \in S^{\prime}$ are placed consecutively in the same circle containing $p$. If these points are placed close enough to $p$, then they will appear consecutively in the colored radial ordering with respect to every point $q_{i}$. The points of $S^{\prime}$ are replaced in the following way: every blue point with a pattern of one red and one blue point; every red point with a pattern of two red and two blue points; every yellow point with a pattern of three red and three blue points; and every green point with a pattern of four red and four blue points. Refer to Figure 3 . Note that our choice of patterns implies that two equivalent radial orderings must match patterns of the same type. So far, $10 \mathrm{~m}$ red and $10 \mathrm{~m}$ blue points have been placed. The remaining $2 r$ points can be placed in such a way that in the radial ordering with respect to every point $q_{i}$ the $r$ red points appear consecutively followed by the $r$ blue points (for example in the line segment joining $p_{1}$ and $p_{2}$. This final condition guarantees that the colored radial orderings at each $q_{i}$ remain different.

To prove the lower bound on $g(n)$, we construct a two-colored set, $P$, of $2 n$ points, in strong general position, with at most $O\left(n^{2}\right)$ colored radial orderings. The construction is somewhat involved. We employ a similar technique as in the proof of Theorem 3.2 We start with a set of $n / 2$ points in strong general position in the unit circle. All the points have the same color and thus the colored radial orderings are all equivalent. Afterwards, we replace each point with a symmetric pattern of red and blue points. This is done in such a way that the new number of distinct colored radial orderings increases to at most $O\left(n^{2}\right)$. 


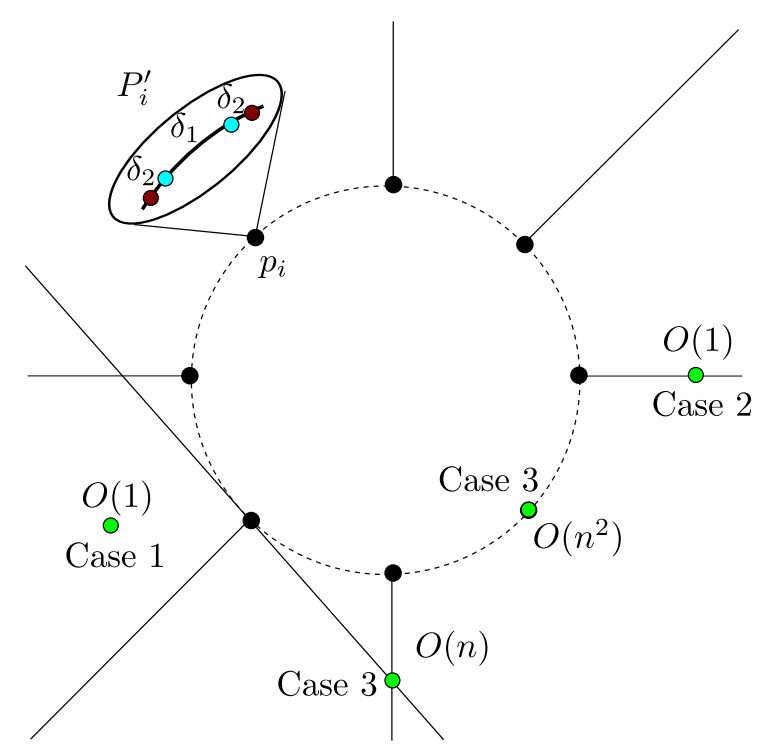

Fig. 4: A bi-colored set with $O\left(n^{2}\right)$ different colored radial orderings.

Before detailing the construction of $P$, we present some definitions and a helpful lemma. A set of lines is in general position if not three lines share a common point and every two lines intersect. Every line $\ell$ in the plane is defined as $\ell:=\{t \in \mathbb{R}: p+t \cdot v\}$, for some point $p$ in the plane and some unit vector $v$ in $\mathbb{R}^{2}$. Define the distance between two lines $\ell_{1}$ and $\ell_{2}$ as the minimum of $\left\|p_{1}-p_{2}\right\|+\left\|v_{1}-v_{2}\right\|$, taken over all pairs $\left(p_{1}, v_{1}\right),\left(p_{2}, v_{2}\right)$ that define $\ell_{1}$ and $\ell_{2}$, respectively. Let $L$ be a set of lines in the plane. For $\delta>0$, a $\delta$-perturbation of $L$ is any set of lines obtained from $L$ by replacing each line by a line at a distance of at most $\delta$. Likewise $S^{\prime}$ is a $\delta$-perturbation of $S$ if it can be obtained from $S$ by replacing each point by a point at a distance of at most $\delta$.

Lemma 3.3 Let $L$ be a set of lines in general position and $L^{\prime}$ a $\delta$-perturbation of $L$. If $\delta$ is small enough then the line arrangement $\mathcal{A}$ generated by $L$ is combinatorially equivalent to the line arrangement $\mathcal{A}^{\prime}$ generated by $L^{\prime}$.

Proof: Let $\ell_{1}$ and $\ell_{2}$ be two lines in $L$ and $\ell_{1}^{\prime}$ and $\ell_{2}^{\prime}$ be the corresponding lines in $L^{\prime}$. Let $p$ be the point of intersection of $\ell_{1}$ and $\ell_{2}$ and let $p^{\prime}$ be the intersection of $\ell_{1}^{\prime}$ and $\ell_{2}^{\prime}$. Note that if $\delta$ is small enough, $p^{\prime}$ is a point. Let $\varepsilon>0$. Choose $\delta$ small enough so that the intersection of any two lines in $L^{\prime}$ is a point, and such that its distance to the corresponding intersection point in $L$ is at most $\varepsilon$.

Now, choose $\varepsilon$ small enough so that the intersection points of any two pairs of lines in $L^{\prime}$ are at a distance greater than 0 . Move each line of $L^{\prime}$ continuously to its corresponding line in $L$, monotonically decreasing their distance throughout the process. Note that since through out the motion not three lines can share a point there are no combinatorial changes in $\mathcal{A}^{\prime}$. Therefore, $\mathcal{A}^{\prime}$ is combinatorially equivalent to $\mathcal{A}$. 

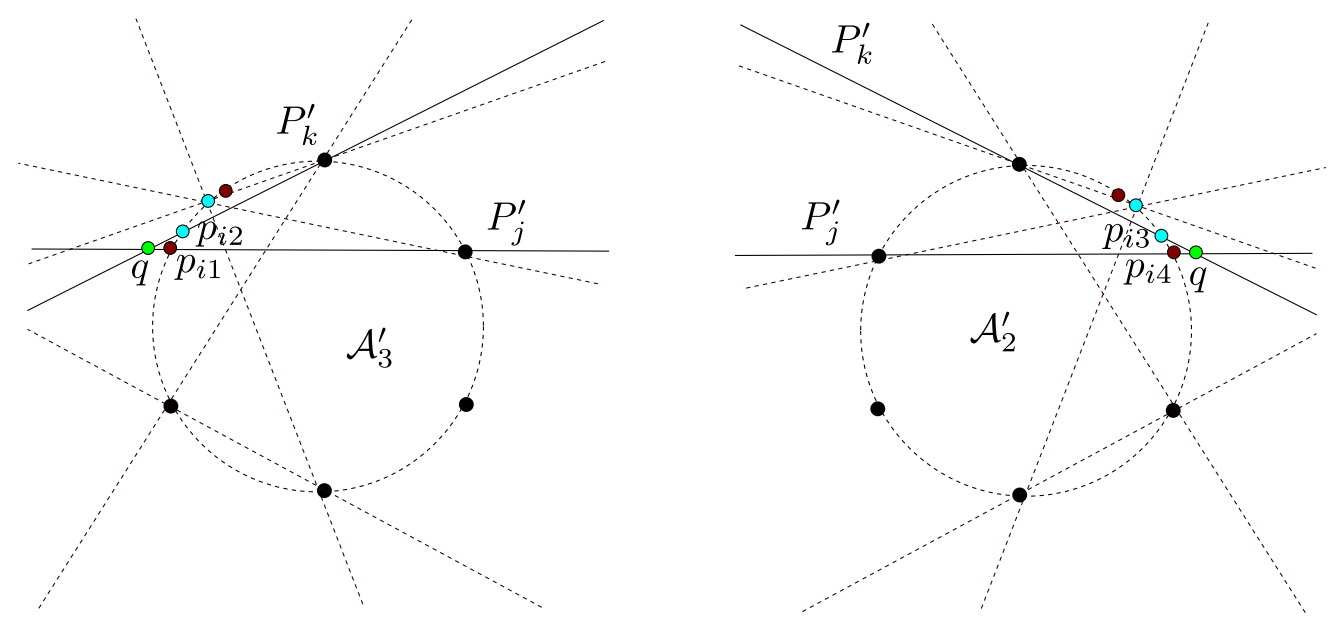

Fig. 5: Schematic depiction of conditions (2) and (3).

We now proceed with the construction of $P$. Let $P^{\prime \prime}$ be a set of $n / 2$ points in strong general position on the unit circle $C$, centered at the origin. Let $L^{\prime \prime}$ be the set of lines passing through every pair of points in $P^{\prime \prime}$, together with the lines passing through every point of $P^{\prime \prime}$ and tangent to $C$. Let $\mathcal{A}^{\prime \prime}$ be the line arrangement generated by $L^{\prime \prime}$.

Let $\delta_{1}, \delta_{2}>0$, such that $\delta_{1}>2 \delta_{2}$. We form a new set of points $P^{\prime}$, by replacing each point $p_{i}$ of $P^{\prime \prime}$ with a set of four points $P_{i}^{\prime}:=\left\{p_{i 1}, p_{i 2}, p_{i 3}, p_{i 4}\right\}$, placed clockwise consecutively in $C$, and colored "red, blue, blue, red" respectively. Place: $p_{i 1}$ at $p ; p_{i 4}$ at distance $\delta_{1}$ from $p_{i 1} ; p_{i 2}$ at distance $\delta_{2}$ from $p_{i 1}$, and $p_{i 3}$ at distance $\delta_{2}$ from $p_{i 4}$. See Figure 4

For $1 \leq k \leq 4$, let $L_{k}^{\prime}$ be the set of lines passing through every pair of points $\left(p_{i k}, p_{j k}\right)$, together with the lines passing through every point of $p_{i k}$ and tangent to $C$. Let $\mathcal{A}_{k}^{\prime}$ be the line arrangement generated by $L_{k}^{\prime}$. Note that $\mathcal{A}^{\prime \prime}, \mathcal{A}_{1}^{\prime}, \mathcal{A}_{2}^{\prime}, \mathcal{A}_{3}^{\prime}$ and $\mathcal{A}_{4}^{\prime}$ are all combinatorially equivalent.

Choose $\delta_{1}$ small enough so that the following condition is met.

(1) Let $C_{1}, C_{2}$ and $C_{3}$ be three closed elements (vertices, edges or cells) in $\mathcal{A}_{i}^{\prime}, \mathcal{A}_{j}^{\prime}$ and $\mathcal{A}_{k}^{\prime}$, respectively. If $C_{1}, C_{2}$ and $C_{3}$ have a non empty intersection, their corresponding elements in $\mathcal{A}^{\prime \prime}$ have a non empty intersection.

Choose $\delta_{2}$ small enough with respect to $\delta_{1}$ so that the following conditions are met:

(2) Let $\ell_{1}$ be a line passing through a point of $P_{j}^{\prime}$ and $p_{i 1}(i \neq j)$. Let $\ell_{2}$ be a line passing through a point of $P_{k}^{\prime}$ and $p_{i 2}(i \neq k$ and $j \neq k)$. If $q$ is the intersection point of $\ell_{1}$ and $\ell_{2}$, then $q$ is in the same cell of $\mathcal{A}_{3}^{\prime}$ as $p_{i 1}$. (See Figure 5 )

(3) Let $\ell_{1}$ be a line passing through a point of $P_{j}^{\prime}$ and $p_{i 4}(i \neq j)$. Let $\ell_{2}$ be a line passing through a point of $P_{k}^{\prime}$ and $p_{i 3}(i \neq k$ and $j \neq k)$. If $q$ is the intersection point of $\ell_{1}$ and $\ell_{2}$, then $q$ is in the same cell of $\mathcal{A}_{2}^{\prime}$ as $p_{i 4}$. (See Figure 5 ) 
Finally, let $P$ be a $\delta_{3}$-perturbation of $P^{\prime}$, with $\delta_{3}<\delta_{2}$, such that $P$ is in strong general position and its elements still belong to $C$. Relabel the points so that $p_{i k}$ is now in $P$. Define $L_{k}, P_{k}$ and $\mathcal{A}_{k}$ with respect to $P$. We choose $\delta_{3}$ small enough so that: Every $\mathcal{A}_{k}$ is combinatorially equivalent to $A^{\prime \prime}$ (Lemma 3.3) and conditions (1), (2) and (3) are still met (with $\mathcal{A}_{2}, \mathcal{A}_{3}, P_{j}, P_{k}$ taking the place of $\mathcal{A}_{2}^{\prime}, \mathcal{A}_{3}^{\prime}, P_{j}^{\prime}, P_{k}^{\prime}$ ).

Theorem 3.4 $g_{\text {col }}(n) \leq O\left(n^{2}\right)$.

Proof: We prove that $P$ has $O\left(n^{2}\right)$ colored radial orderings. Let $q$ be an observation point in the plane. Since there is only one cell of the order partition of $P$ inside its convex hull, we assume that $q$ lies outside the convex hull of $P$. Let $C_{k}$ be the cell containing $q$ in $\mathcal{A}_{k}$. Let $D_{k}$ be the cell corresponding to $C_{k}$ in $\mathcal{A}^{\prime \prime}$. Let $D:=D_{1} \cap D_{2} \cap D_{3} \cap D_{4}$. By condition (1) and Helly's Theorem, $D$ is non-empty.

There are three cases depending on whether $D$ is a cell, an edge or a vertex of $\mathcal{A}^{\prime \prime}$ (see Figure 4):

Case 1. $D$ is a cell. In this case, every pattern replacing a point of $P^{\prime \prime}$ will appear consecutively in the colored radial ordering around $q$. Moreover, by the symmetry of the patterns they are all "red, blue, blue, red". So in this case there is only one possible colored radial ordering.

Case 2. $D$ is an edge. We distinguish two sub-cases, whether the edge is contained in a line passing through two points $p_{i}$ and $p_{j}$ of $P^{\prime \prime}$ or whether it is contained in one of the tangent lines passing through a point $p_{k}$ of $P^{\prime \prime}$.

In the first sub-case, the patterns at points different from $p_{i}$ and $p_{j}$ will appear consecutively in the colored radial ordering (thus each pattern appears as "red, blue, blue, red"). However, the points in the patterns at $p_{i}$ and $p_{j}$ will appear together, but they may be intermixed. Since there are only 8 points involved, there is only a constant number of ways in which this can happen. The second sub-case is similar.

Case 3. $D$ is a vertex. We split the analysis on whether $D$ is in $P^{\prime \prime}$ or not.

- $D$ is a point in $P^{\prime \prime}$. Let $p_{i}$ be the point in $P^{\prime \prime}$ such that $p_{i}$ equals $D$. First suppose that $q$ is neither in the cell of $\mathcal{A}_{3}$ that contains $p_{i 1}$ nor in the cell of $\mathcal{A}_{2}$ that contains $p_{i 4}$. By conditions $(2)$ and (3) in the colored radial ordering with respect to $q$, points $p_{i 1}$ and $p_{i 2}$ appear consecutively or the points between them all belong to some $P_{j}$. Similarly for points $p_{i 3}$ and $p_{i 4}$ and some $P_{k}$. There are at most a constant number of ways in which the points of $P_{j}$ and $P_{k}$ can be intermixed with $\left\{p_{i 1}, p_{i 2}\right\}$ and $\left\{p_{i 3}, p_{i 4}\right\}$ respectively. The patterns replacing other points of $P^{\prime \prime}$ appear consecutively (thus each pattern appears as "red, blue, blue, red"). Summarizing, the points in $\left\{p_{i 1}, p_{i 2}\right\} \cup P_{j}$ appear consecutively as do the points in $\left\{p_{i 3}, p_{i 4}\right\} \cup P_{k}$ and the remaining points appear alternatively as "red, blue, blue, red". There are only $O(n)$ ways in which $\left\{p_{i 1}, p_{i 2}\right\} \cup P_{j}$ can appear with respect to $\left\{p_{i 3}, p_{i 4}\right\} \cup P_{k}$.

Now suppose that $q$ is in the cell of $\mathcal{A}_{2}$ that contains $p_{i 4}$. By conditions (2) and (3) in the colored radial ordering of $P \backslash\left\{p_{i 3}, p_{i 4}\right\}$ with respect to $q, p_{i 1}$ and $p_{i 2}$ appear consecutively; the remaining points of $P \backslash\left\{p_{i 3}, p_{i 4}\right\}$, appear alternatively as "red, blue, blue, red". Thus there are only two possible radial orderings (depending on the position of $p_{i 2}$ with respect to $p_{i 1}$ ) of $P \backslash\left\{p_{i 3}, p_{i 4}\right\}$ with respect to $q$. The only thing remaining to consider are the $O\left(n^{2}\right)$ ways in which $p_{i 3}$ may appear with respect to $p_{i 4}$.

The case where $q$ is in the cell of $\mathcal{A}_{3}$ that contains $p_{i 1}$ is similar to the case where $q$ is in the cell of $\mathcal{A}_{2}$ that contains $p_{i 4}$. In this case there are also at most $O\left(n^{2}\right)$ colored radial orderings. 


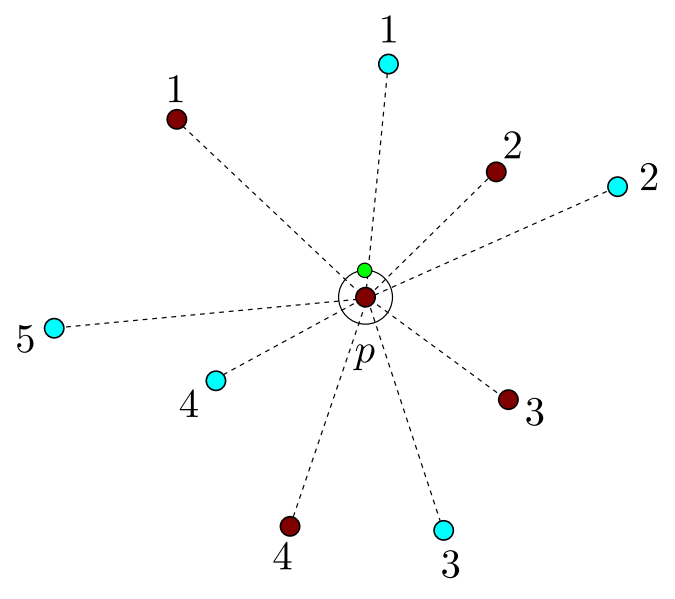

$\sigma_{2}=(1,2,2,3,3, p, 4,4,5,1)$

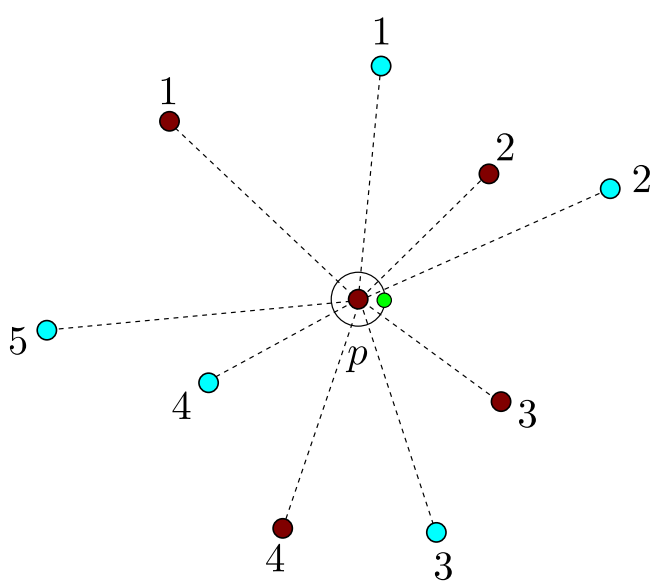

$\sigma_{4}=(1,2,2,3,3,4,4,5, p, 1)$

Fig. 6: A walk showing a linear lower bound on the number of colored radial orderings.

- $D$ is not a point in $P^{\prime \prime}$.

In this case $D$ is the intersection point of two lines $\ell_{1}$ and $\ell_{2}$ in $L^{\prime \prime}$. These lines may be defined by two points or one point of $P^{\prime \prime}$ (depending on whether they are tangent lines or not). In both situations, we have that the patterns at the points of $P^{\prime \prime}$ defining each line appear together but intermixed. The patterns at any other points will appear consecutively (thus each pattern appears as "red, blue, blue, red"). Since there are at most four points defining $\ell_{1}$ and $\ell_{2}$, the number of ways in which their respective pattern points can appear is at most a constant. The only thing left to consider is the $O(n)$ number of ways in which the patterns at the points defining $\ell_{1}$ can appear with respect to those of $\ell_{2}$.

There are at most $O\left(n^{2}\right)$ distinct colored radial orderings in every case.

We now give a linear lower bound for the number of colored radial orderings of $S$. Some notation is required. For a given radial ordering $\sigma$ of $S$, let $\sigma(i)(0 \leq i<2 n)$ be its $(i+1)$ th-element (where addition is taken modulo $2 n$ ). Thus, two radial orderings $\sigma$ and $\rho$ are equivalent whenever there exists a natural number $j$ such that $\sigma(i)=\rho(i+j)$ for all $i$; they are equivalent as colored radial orderings when the color of $\sigma(i)$ is equal to the color of $\rho(i+j)$ for all $i$.

Theorem $3.5 g_{\text {col }}(n) \geq n$.

Proof: Throughout the proof we use both colored and non-colored radial orderings. In each instance we explicitly mention to which of the two types of radial orderings we are referring to. To obtain the claimed lower bound, we show a walk in which $n$ distinct colored radial orderings are seen. First, we choose a red point $p$ of $S$ and let $\mathcal{C}$ be a circle centered at $p$. After that, we walk once clockwise around $\mathcal{C}$. We choose $\mathcal{C}$ to be small enough so that the only half-lines crossed in the walk are those involving $p$ (see Figure 6). 
Consider the non-colored radial orderings seen in this walk. Note that since $\mathcal{C}$ does not cross any halfline defined by points of $S \backslash\{p\}$, the points of $S \backslash\{p\}$ remain fixed in these non-colored radial orderings. The only point that changes position is $p$; it moves counter clockwise, transposing an element of $S \backslash\{p\}$ every time a half-line is crossed. We prove that every time that $p$ transposes a blue point, a new different colored radial ordering is seen.

Since in the walk around $\mathcal{C}$, the blue points of $S$ always appear in the same non-colored radial ordering, we assume that all the non-colored radial orderings of $S$ as seen from points in $\mathcal{C}$ are written starting at the same blue point. Among these radial orderings, for each $k=0, \ldots, n-1$, let $\sigma_{k}$ be the radial ordering in which $p$ is just after the $(k+1)$-th blue point; refer to Figure 6. We show that all the colored radial orderings associated with these $n$ non-colored radial orderings are distinct.

Let then $\sigma_{k}$ and $\sigma_{l}$ be two such non-colored radial orderings encountered at points $q_{1}$ and $q_{2}$ in the walk, respectively. Suppose that $\sigma_{k}$ and $\sigma_{l}$ are equivalent as colored radial orderings. Then there exist a fixed natural number $j$ such that for all $i=0, \ldots, 2 n-1$, the color of $\sigma_{k}(i)$ is equal to the color of $\sigma_{l}(i+j)$.

We now define a directed graph that captures the relationship between $\sigma_{k}$ and $\sigma_{l}$; we employ the structure of this graph to conclude that $k$ must equal $l$. Let $G$ be the directed graph whose vertex set is $S$ and in which, for all $i=0, \ldots, 2 n-1$ there is an arc from $\sigma_{k}(i)$ to $\sigma_{l}(i+j)$; see Figure 7 Note that every vertex in $G$ has indegree and outdegree equal to one. Therefore, $G$ is the union of pairwise disjoint directed cycles of points of the same color.

Let $\Gamma$ be the cycle containing $p$ and let $S^{\prime}:=S \backslash V(\Gamma)$. Let $\rho_{1}$ and $\rho_{2}$ be the non-colored radial orderings of $S^{\prime}$ as seen from $q_{1}$ and $q_{2}$, respectively. We make the additional assumption that $\rho_{1}$ is written starting at $\sigma_{k}(0)$ while $\rho_{2}$ is written starting at $\sigma_{l}(2 n-j)$ (note that being blue, these points are not in $\Gamma)$. Since in particular $p$ is not in $S^{\prime}$, these radial orderings are equivalent. Therefore, there exists a fixed natural number $j^{\prime}$ such that $\rho_{1}(i)=\rho_{2}\left(i+j^{\prime}\right)$, for all $i$. Since $S^{\prime}$ comes from removing the points of $\Gamma$, $\rho_{1}$ can be formed by removing the elements of $\Gamma$ from $\sigma_{k}$ and $\rho_{2}$ can be formed by writing $\sigma_{l}$ starting at $\sigma_{l}(2 n-j)$ and then removing the elements of $\Gamma$ (See Figure 7). Thus the color of $\rho_{1}(i)$ is equal to the color of $\rho_{2}(i)$ for all $i$.

Let $G^{\prime}$ be the directed graph whose vertex set is $S^{\prime}$ and in which there is an arc from $\rho_{1}(i)$ to $\rho_{2}(i)$. As before, every vertex in $G^{\prime}$ has indegree and outdegree equal to one. Therefore $G^{\prime}$ is the union of disjoint cycles of vertices of the same color (in fact $G^{\prime}$ is the subgraph of $G$ induced by $S^{\prime}$ ). Since $\rho_{2}$ is just a "shift" of $j^{\prime}$ places to the right of $\rho_{1}$, all of these cycles have the same length $m$. Therefore, both the number of red and blue points in $S^{\prime}$ are multiples of $m$. This implies that the number of vertices in $\Gamma$ is also a multiple of $m$.

Let $r \cdot m$ be the length of $\Gamma$, since $\Gamma$ is not empty, then $r \geq 1$. Assume that $\Gamma$, starting from $p$ is given by $\left(p=v_{1}, v_{2}, \ldots, v_{m}, \ldots, v_{2 m}, \ldots, v_{r m}\right)$. Let $b_{k}$ be the $(k+1)$-th blue point and $\Gamma^{\prime}:=$ $\left(b_{k}=u_{1}, \ldots, u_{m}\right)$ be the cycle in $G$ containing $b_{k}$. Consider the following sequence of pairs of vertices $\left(u_{1}, v_{1}\right),\left(u_{2}, v_{2}\right), \ldots,\left(u_{m}, v_{m}\right)$. Note that in $\sigma_{k}$, the point $v_{1}=p$ is just after the point $u_{1}=b_{k}$; afterwards, for $2 \leq i \leq m$, the point $v_{i}$ is just after the point $u_{i}$ in both $\sigma_{l}$ and $\sigma_{k}$. (Recall that the order of $S \backslash\{p\}$ in $\sigma_{k}$ and $\sigma_{l}$ is the same.) Suppose that $r>1$, then the point $v_{m+1}$ is just after $u_{1}$ in $\sigma_{l}$ while in $\sigma_{k}$ it is just after the point $v_{1}$ (which is equal to $p$ ). Consider now the following sequence of vertices $\left(v_{1}, v_{m+1}\right),\left(v_{2}, v_{m+2}\right), \ldots,\left(v_{(r-1) m+1}, v_{r m+1}=p\right)$. From the same arguments as before, for $m+2 \leq i \leq r m$, the point $v_{i}$ is just after the point $v_{i-m}$ in both $\sigma_{k}$ and $\sigma_{l}$. For $i=r m+1$, the point $v_{r m+1}$ is just after the point $v_{(r-1) m+1}$ in $\sigma_{l}$, but $v_{r m+1}=p$ and $v_{(r-1) m+1}$ is red, a contradiction since $p$ is just after a blue point in $\sigma_{l}$. Thus $r=1$. This implies that $\sigma_{k}=\sigma_{l}$, since $v_{1}$ (which is equal to $p$ ) is 

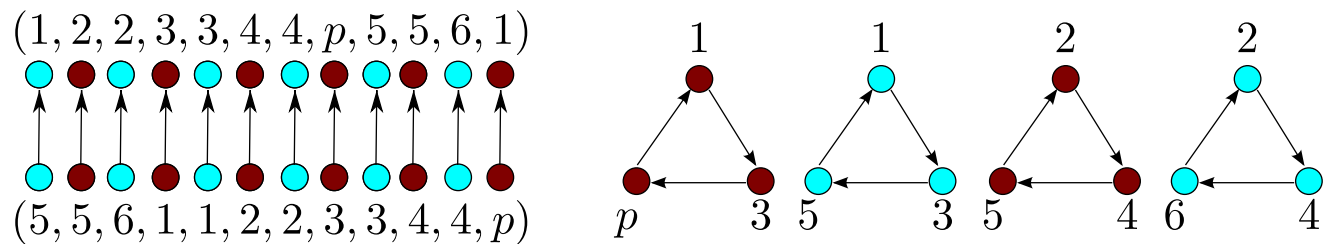

Fig. 7: Two equivalent colored radial orderings and their corresponding graph.

after $u_{1}$ (which is equal to $b_{k}$ ) in both $\sigma_{l}$ and $\sigma_{k}$.

\section{Conclusions}

We proved an upper bound of $O\left(n^{4}\right)$ and a lower bound of $\Omega\left(n^{3}\right)$ on the number of radial orderings that every set of $n$ points in strong general position in the plane must have. The upper bound was first given in [7]. As a corollary in the same paper it was noted that every set of $n$ points in the plane contains $O\left(n^{4}\right)$ different star shaped polygonizations. Our result implies that every set of $n$ points in strong general position in the plane has $\Omega\left(n^{3}\right)$ different star shaped polygonizations. We leave the closing of this gap as an open problem:

Conjecture 4.1 $g(n)=\Theta\left(n^{4}\right)$.

We used the assumption that $S$ is in strong general position heavily on the proof of the lower bound on $g(n)$. However, we believe that it is not needed and that only general position (no three collinear points) is sufficient.

Conjecture 4.2 If $S$ is a set of $n$ points in general position in the plane, then it has at least $\Omega\left(n^{4}\right)$ distinct radial orderings.

For colored point sets the situation is far more intriguing, here we have been able to prove that such a gap exists. Mainly that there are bi-colored sets of $2 n$ points with $\Theta\left(n^{4}\right)$ colored radial orderings and sets with only $\Theta\left(n^{2}\right)$. The best lower bound we have been able to provide is of $\Omega(n)$. We make the following conjecture.

Conjecture 4.3 $g_{\mathrm{col}}(n)=\Theta\left(n^{2}\right)$.

Note that we used the assumption that $n$ is even heavily in the proof of Theorem 3.4. In fact, it can be shown that the number of colored radial orderings may increase to $\Theta\left(n^{3}\right)$ if a red and a blue point are added to the construction in the proof of Theorem 3.4 Also in the proof of Theorem 3.5 we relied on the fact that the number of red points equals the number of blue points. It is possible to construct a set of $n$ red and $n-1$ blue points such that a walk like the one described in Theorem 3.5 yields only one colored radial ordering. It may be the case that the bounds given in Theorems 3.4 and 3.5 no longer hold when either one of these two hypothesis is dropped.

Acknowledgments. The problems studied here, were introduced and partially solved during a stay at Universidad de la Habana, Cuba, January 2010. The authors would like to thank our host Carlos Ochoa during this visit, and Clemens Huemer; Merce Claverol and David Wood for helpful comments. 


\section{References}

[1] B. M. Ábrego and S. Fernández-Merchant. A lower bound for the rectilinear crossing number. Graphs Combin., 21(3):293-300, 2005.

[2] J. Balogh and G. Salazar. $k$-sets, convex quadrilaterals, and the rectilinear crossing number of $K_{n}$. Discrete \& Comput. Geom., 35(4):671-690, 2006.

[3] I. Bárány and I. Matoušek. Equipartition of two measures by a 4-fan. Discrete \& Comput. Geom., 27:293-301, 2002.

[4] I. Bárány and J. Matoušek. Simultaneous partitions of measures by $k$-fans. Discrete \& Comput. Geom., 25:317334, 2001.

[5] S. Bereg. Equipartitions of measures by 2-fans. Discrete \& Comput. Geom., 34:87-96, 2005.

[6] S. Bespamyatnikh, D. Kirkpatrick, and J. Snoeyink. Generalizing ham-sandwich cuts to equitable subdivisions. Discrete \& Comput. Geom., 24:605-622, 2000.

[7] L. Deneen and G. Shute. Polygonizations of point sets in the plane. Discrete \& Comput. Geom., 3(1):77-87, 1988.

[8] O. Devillers, V. Dujmović, H. Everett, S. Hornus, S. Whitesides, and S. Wismath. Maintaining visibility information of planar point sets with a moving viewpoint. Internat. J. Comput. Geom. Appl., 17(4):297-304, 2007.

[9] J. M. Díaz-Bañez, R Fabila-Monroy, and P. Pérez-Lantero. On the number of radial orderings of colored planar point sets. In Computational Geometry (XIV Spanish Meeting on Computational Geometry), volume 7579 of LNCS, pages 126-137, Alcalá de Henares, Spain, 2011.

[10] B. Gfeller, M. Mihalák, S. Suri, E. Vicari, and P. Widmayer. Counting targets with mobile sensors in an unknown environment. In Proceedings of the 3rd international conference on Algorithmic aspects of wireless sensor networks, ALGOSENSORS'07, pages 32-45, Berlin, Heidelberg, 2008. Springer-Verlag.

[11] O. Kannianen and T. M. R. Allho. Minimalist navigation for a mobile robot based on a simple visibility sensor information. In AI and Machine Consciousness, Proceedings of the 13th Finnish Artificial Intelligence Conference STeP 2008, pages 68-75, 2008.

[12] S. Langerman and Steiger W. Optimization in arrangements. In In Proc. 20th Sympos. Theoret. Aspects Comput. Sci., Lect. Notes in Comput. Sci., vol. 2607, pages 50-61, Berlin, Heidelberg, 2003. Springer-Verlag.

[13] L. Lovász, K. Vesztergombi, Uli Wagner, and Emo Welzl. Convex quadrilaterals and $k$-sets. In Towards a theory of geometric graphs, volume 342 of Contemp. Math., pages 139-148. Amer. Math. Soc., Providence, RI, 2004.

[14] S. Suri, E. Vicari, and P. Widmayer. Simple robots with minimal sensing: From local visibility to global geometry. Int. J. Rob. Res., 27:1055-1067, September 2008.

[15] B. Tovar, L. Freda, and S. M. LaValle. Using a robot to learn geometric information from permutations of landmarks. In Topology and robotics, volume 438 of Contemp. Math., pages 33-45. Amer. Math. Soc., Providence, RI, 2007.

[16] R. Ulber. On the number of star-shaped polygons and polyhedra. In Proc. 11th Canadian Conference on Computational Geometry CCCG'99, Vancouver, Canada, 1999. 